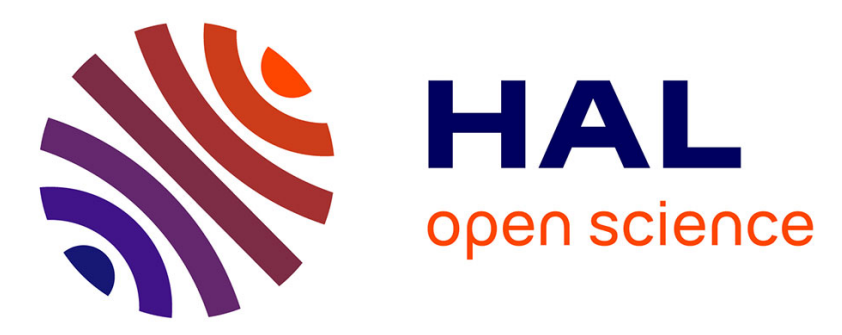

\title{
Reconstitution d'une impulsion lumineuse brève. Étude des fluctuations temporelles d'un photomultiplicateur
}

\author{
J.A. Miehe, B. Sipp
}

\section{To cite this version:}

J.A. Miehe, B. Sipp. Reconstitution d'une impulsion lumineuse brève. Étude des fluctuations temporelles d'un photomultiplicateur. Revue de Physique Appliquée, 1975, 10 (3), pp.127-135. 10.1051/rphysap:01975001003012700 . jpa-00243891

\section{HAL Id: jpa-00243891 https://hal.science/jpa-00243891}

Submitted on 1 Jan 1975

HAL is a multi-disciplinary open access archive for the deposit and dissemination of scientific research documents, whether they are published or not. The documents may come from teaching and research institutions in France or abroad, or from public or private research centers.
L'archive ouverte pluridisciplinaire HAL, est destinée au dépôt et à la diffusion de documents scientifiques de niveau recherche, publiés ou non, émanant des établissements d'enseignement et de recherche français ou étrangers, des laboratoires publics ou privés. 
Classification

Physics Abstracts

0.676

\title{
RECONSTITUTION D'UNE IMPULSION LUMINEUSE BRÈVE. ÉTUDE DES FLUCTUATIONS TEMPORELLES D'UN PHOTOMULTIPLICATEUR
}

\author{
J. A. MIEHE et B. SIPP \\ Laboratoire de Physique des Rayonnements et d'Electronique Nucléaire, \\ Centre de Recherches Nucléaires et Université Louis-Pasteur \\ 67037 Strasbourg Cedex, France
}

(Reçu le 5 septembre 1974, révisé le 6 janvier 1975, accepté le 21 janvier 1975)

\begin{abstract}
Résumé. - Dans le cadre d'une mise au point consacrée à l'étude des fluctuations temporelles intervenant dans les photomultiplicateurs à grand gain de première dynode utilisés dans les conditions de photoélectron unique, on rappelle brièvement l'analyse théorique de la méthode de reconstitution d'une impulsion lumineuse et on passe en revue les résultats expérimentaux concernant la dispersion temporelle due à l'optique d'entrée. Cette étude a été prolongée en examinant l'influence des variations du temps de transit dans le multiplicateur sur les courbes de résolution en temps du détecteur : on montre que la largeur de celles-ci dépend du seuil du discriminateur qui définit l'instant d'apparition du signal anodique. Dans les conditions de faibles niveaux de déclenchement, on a mesuré une largeur à mi-hauteur de la fonction de réponse égale à 270 ps.
\end{abstract}

\begin{abstract}
This paper reviews the results on time fluctuations in high gain first dynode photomultipliers used in single photon timing experiments; we recall the theoretical analysis of the measurement of the shape of light pulses and we discuss previously obtained results concerning time dispersion in the photocathode - first dynode space. In addition, we examine the influence of the variations of the electron transit time in the multiplier on the time resolution curves of the detector : the curves obtained by leading-edge triggering of the anodic pulse show a strong dependence on the threshold level of the discriminator. A single-photoelectron timing resolution of $270 \mathrm{ps}$ is measured using a low leading edge discrimination.
\end{abstract}

1. Introduction. - La reconstitution d'impulsions lumineuses fondée sur les propriétés statistiques des instants d'émission des photoélectrons est une technique employée avec succès pour mesurer les durées de vie de niveaux atomiques excités [1] et pour déterminer la loi de déclin de la fluorescence [2] ou de la radioluminescence [3] de systèmes moléculaires. La distribution expérimentale $F(t)$ obtenue par cette méthode est la convolution de deux fonctions : l'une, $i(t)$, représente la variation de l'intensité lumineuse avec le temps (fonction d'illumination), la seconde, $H(t)$, est la fonction de réponse du dispositif expérimental. Cette méthode jointe à l'utilisation d'éclairs lumineux bref (de largeur inférieure à la nanoseconde) a permis d'étudier les performances temporelles des photomultiplicateurs : mesures de la dispersion du temps de transit d'un photoélectron dans l'espace cathode-première dynode $[4,5]$, de la résolution en temps et de la réponse percussionnelle en photoélectron unique $[6,7]$.

Récemment, Poultney [8] ainsi que Cova et coll. [9] ont publié une mise au point concernant la détermination de la courbe de décroissance de l'intensité lumi- neuse par détection de photons uniques et les méthodes d'exploitation des résultats expérimentaux. Par contre, l'influence des fluctuations temporelles introduites par le photomultiplicateur utilisé en vue de reproduire des impulsions lumineuses très brèves n'a pas été discutée.

Dans un travail précédent, nous avions montré [10] que l'émission des électrons par une photocathode soumise à des éclairs lumineux peut être décrite par les lois de probabilité des intervalles de temps séparant les instants d'apparition du signal et d'émission du premier photoélectron. Nous avions également déterminé expérimentalement les distributions temporelles des photoélectrons au moyen d'un photomultiplicateur à grand gain de première dynode dont l'intérêt est de pouvoir discerner les événements qui donnent naissance à un, deux, ... photoélectrons.

Dans ce travail, nous avons entrepris une étude expérimentale des différentes contributions à l'élargissement de la fonction de réponse du détecteur. Nous rappellerons d'abord les lois donnant la probabilité des instants d'émission des photoélectrons et présenterons les résultats ainsi que les conclusions auxquelles ils 
conduisent dans l'hypothèse d'une répartition poissonnienne du nombre de photoélectrons. Enfin, nous déterminerons les diverses fluctuations liées à l'instant d'apparition du signal anodique et nous discuterons l'influence de la dispersion introduite par le multiplicateur d'électrons sur la réponse temporelle en photoélectron unique.

2. Lois de probabilité des instants d'émission des photoélectrons en régime impulsionnel. - Lorsque la cathode d'un photomultiplicateur est soumise à un flux lumineux d'intensité suffisamment faible, les instants d'émission des photoélectrons forment une suite d'événements aléatoires appelée processus ponctuel. Les lois de probabilité auxquelles obéit ce dernier, ne sont accessibles expérimentalement que par comptage du nombre d'impulsions ou par la détermination de la distribution des intervalles de temps séparant deux photoélectrons consécutifs. Les caractéristiques de ce processus ponctuel ont été étudiées par de nombreux auteurs [11, 12] qui ont montré que ses propriétés peuvent s'interpréter par une loi de Poisson composée.

Pour une photocathode éclairée par une source d'impulsions lumineuses dont la loi de variation avec le temps est $N_{0} i(t), N_{0}$ représentant l'espérance mathématique du nombre de photons émis (distribution de Poisson) au cours de l'éclair lumineux et $i(t) \mathrm{d} t$ la probabilité de l'instant d'apparition d'un photon dans l'intervalle de temps $(t, t+\mathrm{d} t)$, le nombre de photoélectrons $n$ enregistrés entre les instants $t_{1}$ et $t_{2}$ obéit à une loi de Poisson non uniforme donnée par la relation :

$$
\begin{aligned}
\operatorname{Pr}\left[n\left(t_{1}, t_{2}\right)=k\right]= & \frac{\left[\alpha N_{0} I\left(t_{1}, t_{2}\right)\right]^{k}}{k !} \times \\
& \times \exp \left[-\alpha N_{0} I\left(t_{1}, t_{2}\right)\right]
\end{aligned}
$$

$I\left(t_{1}, t_{2}\right)$ désignant l'intégrale $\int_{t_{1}}^{t_{2}} i(u) \mathrm{d} u$ et $\alpha$ le rendement quantique de la photocathode.

La suite des photoélectrons émis peut d'autre part être caractérisée par la distribution $R_{k}(t)$ des intervalles de temps $T$ égaux à $T_{k}-T_{i}, T_{k}$ désignant la date d'émission du $k$-ième photoélectron et $T_{i}$ celle d'un signal synchrone de l'impulsion lumineuse ; en considérant que l'instant $T_{i}$ est connu rigoureusement, on a :

$$
\begin{aligned}
& R_{k}(t)=\alpha N_{0} i(t) \frac{\left[\alpha N_{0} I(0, t)\right]^{k-1}}{(k-1) !} \\
& \times \exp \left[-\alpha N_{0} I(0, t)\right]
\end{aligned}
$$

2.1 LOI DE PROBABILITÉ DES INSTANTS D'ÉMISSION DU PREMIER PHOTOÉLECTRON. - Dans ce cas, la relation précédente s'écrit :

$$
R_{1}(t)=\alpha N_{0} i(t) \exp \left[-\alpha N_{0} I(0, t)\right] .
$$

Lorsque le nombre moyen de photoélectrons créés est faible $\left(\alpha N_{0}<0,01\right)$, les expressions précédentes peuvent se mettre sous la forme suivante :

$$
\begin{gathered}
\operatorname{Pr}[n(0, t)=1] \approx \alpha N_{0} I(0, t) \\
\operatorname{Pr}[n(0, t)=2] \approx \frac{\left[\alpha N_{0} I(0, t)\right]^{2}}{2} \approx 0 \\
R_{1}(t) \approx \alpha N_{0} i(t) .
\end{gathered}
$$

Dans les conditions d'un faible éclairement de la photocathode (photoélectron unique), on peut conclure que :

- le nombre d'événements détectés est une variable aléatoire de pile ou face et dont la valeur moyenne est proportionnelle à l'intensité lumineuse ;

- la distribution des instants d'émission du photoélectron est identique à la fonction d'illumination $i(t)$.

Bollinger et Thomas [13], Koechlin [14] et Pfeffer [15] ont utilisé les critères précédents pour déterminer la loi de décroissance de la radioluminescence des scintillateurs en enregistrant avec un convertisseur temps-amplitude l'histogramme des intervalles de temps séparant les instants d'arrivée de l'impulsion anodique d'un photomultiplicateur et d'un signal synchrone de l'excitation.

De la relation (3) donnant $R_{1}(t)$, on déduit par intégration que pour un nombre de photoélectrons $\alpha N_{0}$, la fonction d'illumination $i(t)$ vérifie l'équation :

$$
i(t)=\frac{1}{\alpha N_{0}} \cdot \frac{R_{1}(t)}{1-\int_{0}^{t} R_{1}(u) \mathrm{d} u} .
$$

La connaissance expérimentale de $R_{1}(t)$ permet de calculer $i(t)$; il est à noter que la relation (3) s'écrit :

$$
\begin{aligned}
R_{1}(t) & =-\frac{\mathrm{d}}{\mathrm{d} t}\left\{\exp \left[-\alpha N_{0} I(0, t)\right]\right\} \\
& =-\frac{\mathrm{d}}{\mathrm{d} t}\{\operatorname{Pr}[n(0, t)=0]\}
\end{aligned}
$$

La loi de probabilité de l'instant d'émission du premier photoélectron est proportionnelle à la dérivée de la probabilité de ne trouver aucun photoélectron dans l'intervalle $(0, t)$.

2.2 DisTRIBUTIONS DES INSTANTS D'ÉMISSION DU PREMIER PHOTOÉLECTRON POUR UN NOMBRE DÉTERMINÉ DE PHOTOÉLECTRONS ÉMIS PAR IMPULSION LUMINEUSE. Ces distributions de probabilité présentent un intérêt pratique lorsque le détecteur utilisé est un photomultiplicateur à grand gain de première dynode (RCA 8850). Nous rappelons que pour un tel tube, le spectre d'amplitude présente des maximums correspondant à l'émission de $1,2, \ldots$ photoélectrons (Fig. 1). Il est donc possible d'obtenir expérimentalement les distributions $r_{k}(t)$ de l'instant d'émission du premier électron lorsque $k$ photoélectrons sont émis par éclair lumineux. 


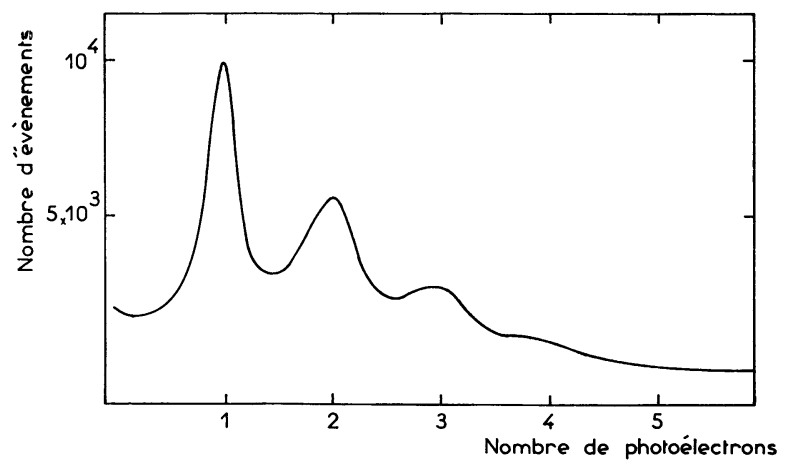

Fig. 1. - Spectre des hauteurs d'impulsions d'un photomultiplicateur à grand gain de première dynode dans le cas d'un éclairement faible de la photocathode.

Compte tenu des relations (1) et (2), on a :

$r_{k}(t)=\alpha N_{0}\left[\exp \left(-\alpha N_{0}\right)\right] i(t) \frac{\left[\alpha N_{0}(1-I(0, t))\right]^{k-1}}{(k-1) !}$

Dans les conditions où un seul photoélectron est créé par impulsion lumineuse, on a :

$$
r_{1}(t)=\alpha N_{0}\left[\exp \left(-\alpha N_{0}\right)\right] i(t) .
$$

Cette relation est vérifiée quel que soit le nombre de photons incidents $N_{0}$. La probabilité $p_{1}$ d'observer un événement à un seul photoélectron est proportionnelle à $\alpha N_{0} \exp \left(-\alpha N_{0}\right)$; elle est maximum lorsque $\alpha N_{0}$ est égal à l'unité. Dans ce dernier cas, la durée d'une expérience de reconstitution de la fonction d'illumination $i(t)$ est notablement réduite ; en effet, la fréquence relative de détection $p_{1}$ d'un événement est égale à $1 / e$, elle est inférieure à $1 \%$ dans les conditions de photoélectron unique.

2.3 DisPositiF DE MESURE ET RÉSULTATS EXPÉRIMENTAUX. - Le montage expérimental, représenté schématiquement sur la figure 2 se compose d'un photomultiplicateur RCA 8850 et d'un dispositif de coïncidences retardées lent-rapide. La source lumineuse éclairant faiblement la photocathode est constituée par une diode électroluminescente au phosphure de gallium (FERRANTI XP 22). Un éclair lumineux est obtenu en appliquant à la diode des impulsions électriques d'amplitude égale à $10 \mathrm{~V}$ et de largeur à mi-hauteur voisine de 15 ns. A l'aide d'un convertisseur tempsamplitude (CTA), on établit l'histogramme des intervalles de temps séparant les instants d'apparition de l'impulsion électrique (synchrone de l'éclair lumineux) et du signal anodique correspondant à l'émission du premier photoélectron. Le sélecteur d'amplitude SA permet de choisir les événements qui correspondent à un nombre déterminé de photoélectrons.

Sur la figure 3 sont reportées les courbes obtenues $r_{1}(t)$ et $r_{2}(t)$; la courbe en traits pleins, calculée par l'intermédiaire de la relation (8) et des valeurs numériques de $r_{1}(t)$, est en excellent accord avec les résultats

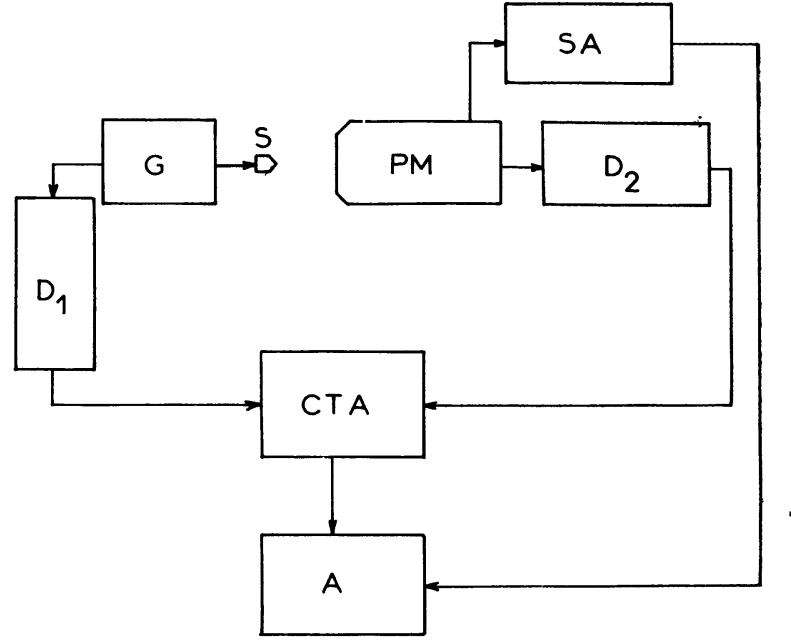

$S$ Source lumineuse

PM Photomultiplicateur

$D_{1} \& D_{2}$ Discriminateurs

SA Sélecteur d'amplitude

C TA Convertisseur remps amplitude

$G \quad$ Générateur d'impulsions

A Analyseur dámplitude

FIG. 2. - Dispositif de coïncidences retardées.

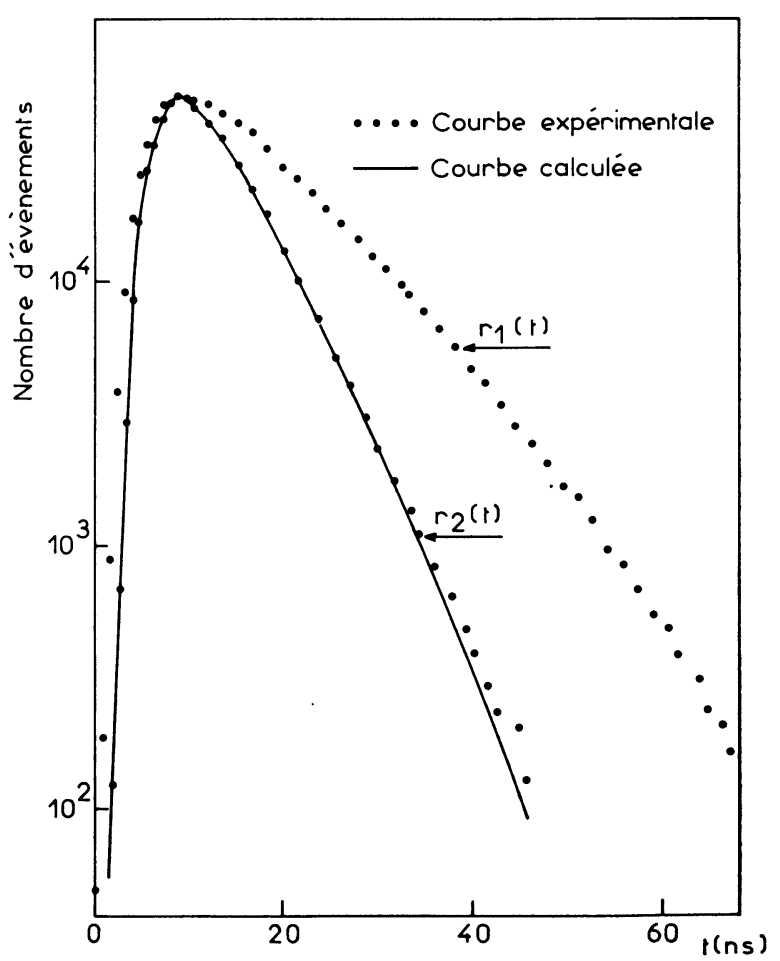

Fig. 3. - Histogrammes des intervalles de temps $r_{1}(t)$ et $r_{2}(t)$.

expérimentaux, ce qui montre l'intérêt du pouvoir de résolution en amplitude élevé de ce type de compteur pour l'étude des distributions temporelles des photoélectrons.

3. Fluctuations temporelles liées au photomultiplicateur. - Dans les expériences de reconstitution 
d'éclairs lumineux brefs (domaine de la sub-nanoseconde), les fluctuations temporelles de l'impulsion anodique du photomultiplicateur ne peuvent plus être négligées par rapport à celles des instants d'émission des photoélectrons et par suite, l'expression de la distribution des intervalles de temps $r_{1}(t)$ est modifiée.

L'origine des fluctuations de l'instant d'apparition du signal anodique consécutif à l'émission d'un seul photoélectron est de nature différente suivant qu'elles prennent naissance dans l'espace photocathode-première dynode ou qu'elles proviennent du système multiplicateur d'électrons. La première composante est due à la fois aux différences de trajets des électrons émis en des points différents de la photocathode (écarts centrebord) [16] et aux variations de leurs vitesses initiales [17]. Les fluctuations introduites par le multiplicateur résultent du caractère statistique du processus d'émission secondaire et de la dispersion des temps de transit des électrons entre deux dynodes successives. Dans ces conditions, la réponse $f(t)$ du photomultiplicateur peut donc être mise sous la forme :

$$
f(t)=k(t) * m(t)
$$

et la distribution expérimentale $r_{1 \text { exp }}(t)$ s'écrit :

$$
r_{1 \exp }(t)=r_{1}(t) * k(t) * m(t)
$$

$k(t)$ et $m(t)$ décrivant respectivement les densités de probabilité du temps de vol d'un photoélectron dans l'optique d'entrée et de l'instant d'apparition du signal anodique lorsque un seul photoélectron arrive sur la première dynode. Les caractéristiques de la distribution $m(t)$ dépendent du mode d'obtention d'une information temporelle à partir de l'impulsion anodique (front de montée, passage par zéro) et font intervenir le mode de collection des charges à l'anode (impulsion de courant ou de charge). Les propriétés de $k(t)$ sont déterminées par la structure géométrique de l'optique d'entrée du photomultiplicateur.

3.1 DISPERSION DU TEMPS DE TRANSIT DES PHOTOÉLECTRONS. - Les écarts entre les temps de vol correspondant aux effets centre-bord peuvent être sensiblement diminués par un éclairement ponctuel de la photocathode. L'influence des variations des vitesses initiales des électrons sur le temps de transit est alors prépondérante ; elle fait intervenir :

- la composante tangentielle qui n'affecte pas au premier ordre le temps de transit, mais change le point d'impact d'un photoélectron sur la première dynode [16] ;

- la composante normale qui modifie le temps de vol de la photocathode à la première dynode ; pour deux photoélectrons émis normalement à la photocathode avec une différence d'énergie $\Delta E$, l'écart $\Delta t$ est donné par [17] :

$$
\Delta t \approx \sqrt{\frac{2 m \Delta E}{e}} \cdot \frac{1}{\mathcal{E}}
$$

$\mathcal{E}$ étant le champ électrique sur la photocathode, $e$ et $m$ la charge et la masse de l'électron.

Cette dernière contribution a été mise en évidence par des expériences de reconstitution de forme d'un éclair lumineux bref. Les histogrammes $r_{1 \exp }(t)$ de la figure 4 ont été relevés [18] pour différentes valeurs du champ électrique sur la cathode d'un photomultiplicateur RCA 8850 à grand gain de première dynode ; les tensions appliquées à l'optique d'entrée sont respec-

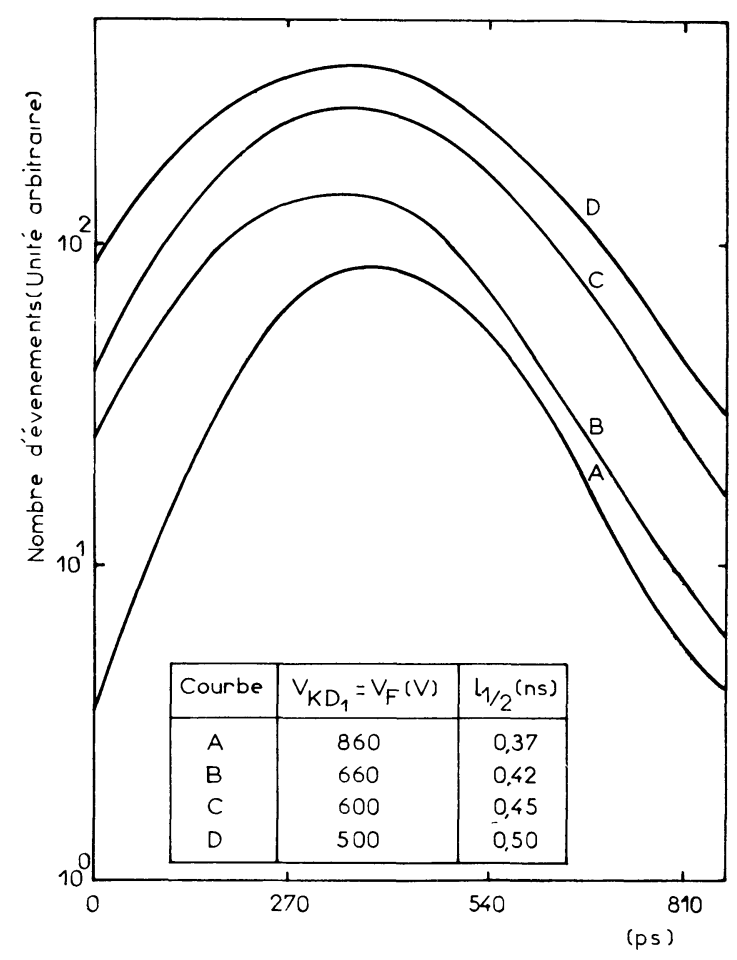

FIG. 4. - Influence de la variation de la tension photocathode première dynode sur les distributions $r_{1 \exp }(t)$.

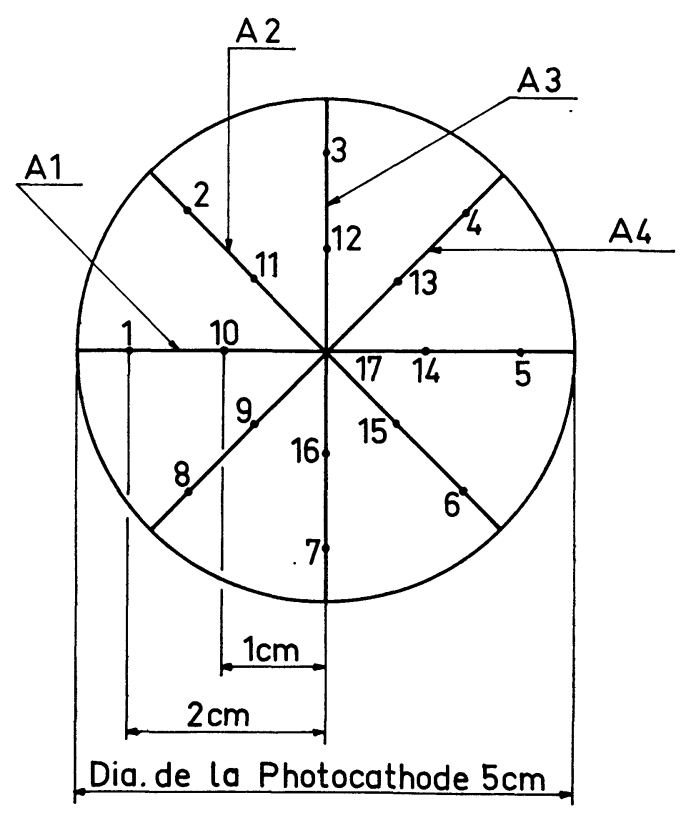

FIG. 5. - Points d'émission des électrons`sur la photocathode. 
tivement égales à $860 \mathrm{~V}, 660 \mathrm{~V}, 600 \mathrm{~V}$ et $500 \mathrm{~V}$. On note une augmentation de l'écart-type du temps de vol égale à $100 \mathrm{ps}$ lorsque la tension de la première dynode varie de $860 \mathrm{~V}$ à $660 \mathrm{~V}$.

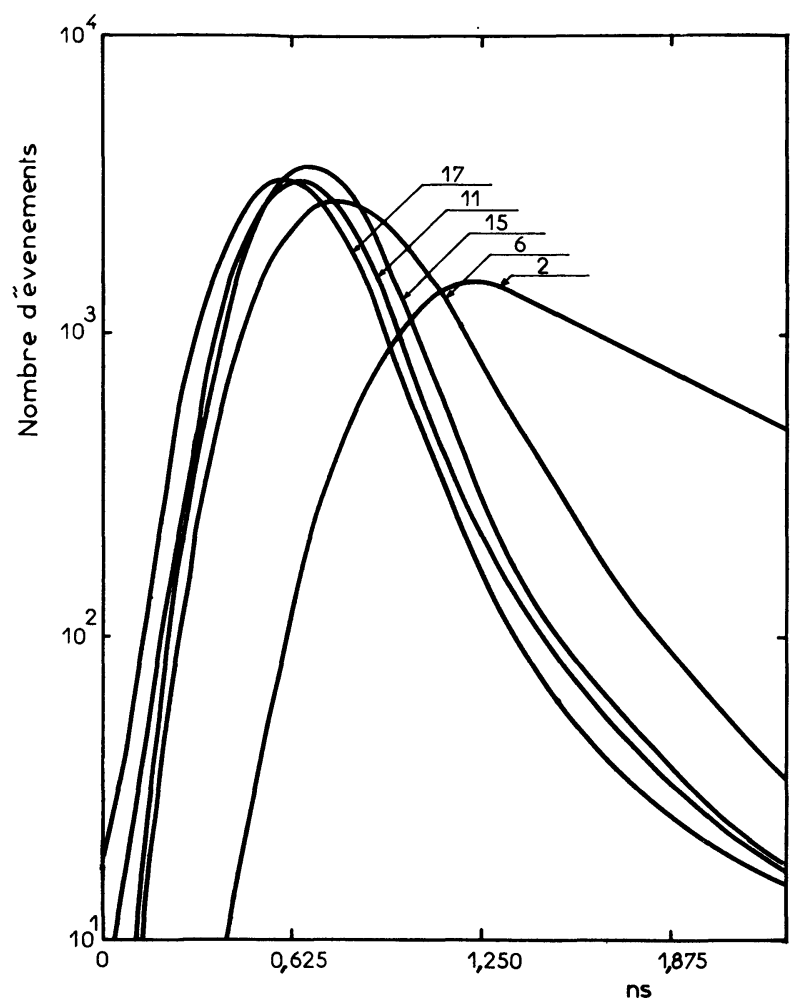

FIG. $6 a$. - Distributions $r_{1 \exp }(t)$ correspondant à différents points d'émission situés sur l'axe $A_{2}$.

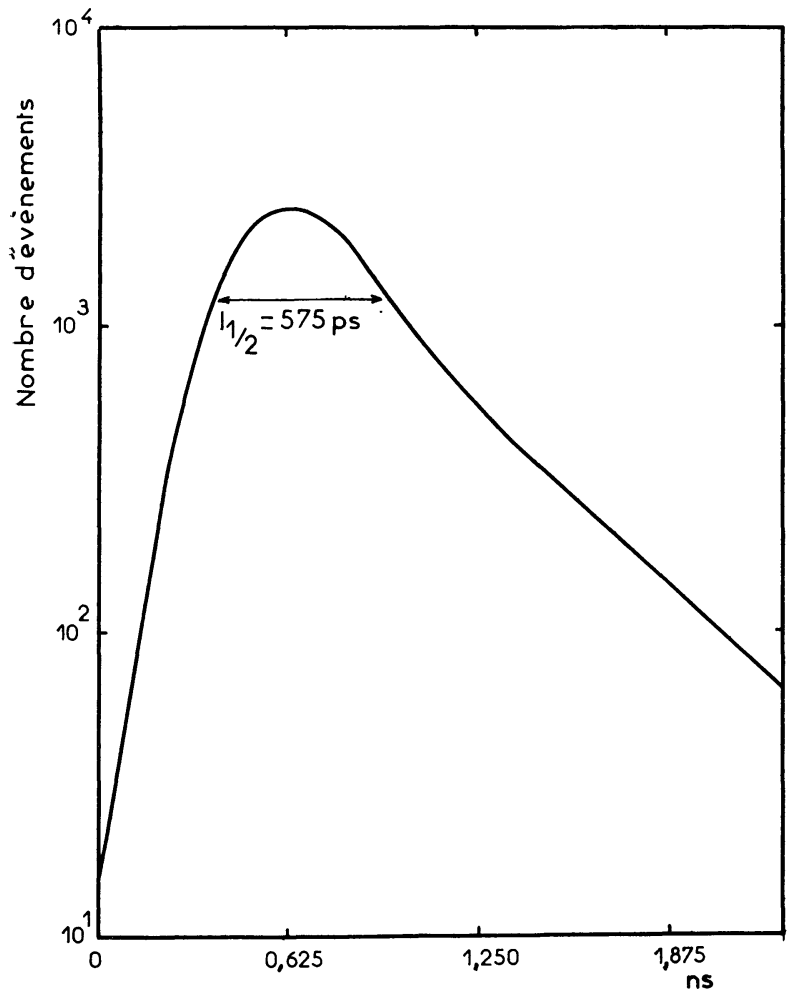

FIG. $6 b .-$ Distributions $r_{1 \exp }(t)$ correspondant à un éclairement uniforme de la photocathode.
Les écarts centre-bord constituent une des causes principales des variations du temps de transit des photoélectrons : pour deux électrons émis simultanément et sans vitesse initiale, l'écart $\Delta t$ dû à une différence de parcours $\Delta x$ est :

$$
\Delta t=\Delta x \sqrt{\frac{m}{2 e V}}
$$

$V$ étant la différence de potentiel appliquée. Pour les photomultiplicateurs à optique d'entrée comportant des électrodes de focalisation et d'accélération, cet écart est estimé à 100 ps à une distance égale à $2 \mathrm{~cm} \mathrm{du}$ centre de la cathode.

Les écarts centre-bord ont été déterminés pour un photomultiplicateur RCA 8850 [19] en enregistrant pour un éclairement ponctuel et pour différents points de la cathode (Fig. 5) les distributions $r_{1 \exp }(t)$. Les courbes obtenues (Fig. 6a) correspondent aux points situés sur le diamètre $A_{2}$. Les variations des différences des temps de transit moyens $T$ ainsi que celles des largeurs à mi-hauteur $l_{1 / 2}$, en fonction des points de la cathode, sont reportées sur les figures $7 a$ et $7 b$.

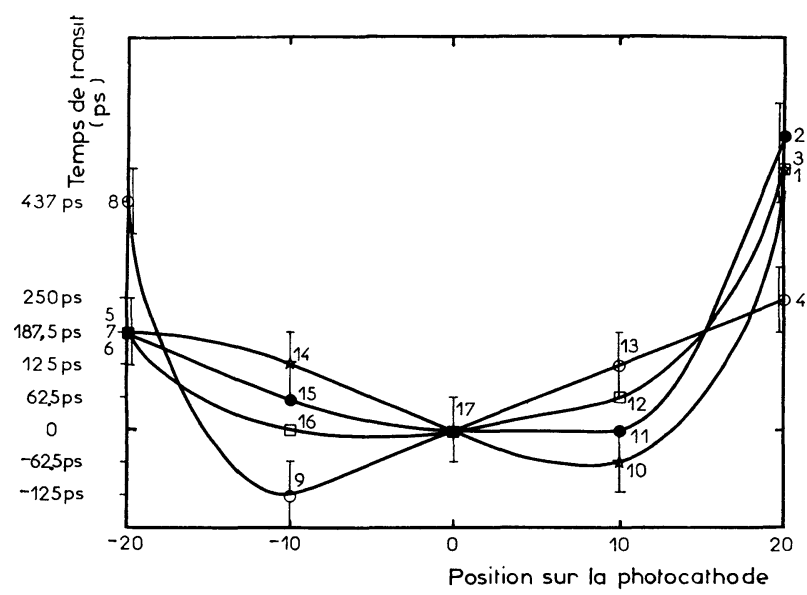

FIG. 7a. - Courbes de variation du temps de transit en fonction des points d'émission de la photocathode.

$11 / 2$ (ps)

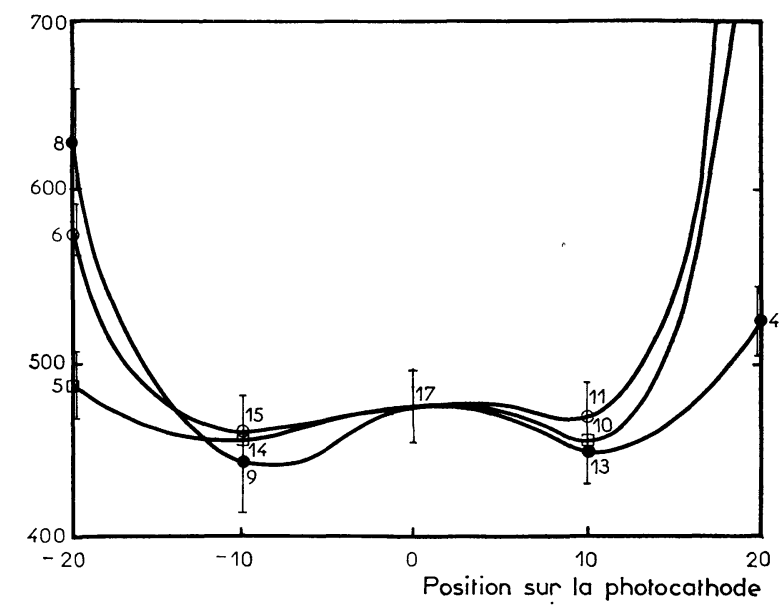

FIG. 7b. - Courbes de variation de la largeur à mi-hauteur des distributions $r_{1 \exp }(t)$ avec la position d'éclairement de la photocathode. 
De l'examen des résultats expérimentaux, on peut conclure que :

- dans un rayon d'un centimètre, les différences des temps de vol sont inférieures à $125 \mathrm{ps}$; les courbes de variation des temps de transit (diamètre $A_{1}$ et $A_{4}$ ) présentent une asymétrie due à l'inclinaison de la première dynode par rapport à l'axe de l'optique d'entrée, par contre celles correspondant aux points situés sur les diamètres $A_{2}$ et $A_{3}$, parallèles à la génératrice de la dynode, sont symétriques ;

- les courbes expérimentales (Fig. 6a) mettent en évidence des variations importantes de la forme des distributions du temps de vol suivant les points d'émission des photoélectrons : ces résultats sont en désaccord avec l'hypothèse d'une répartition uniforme $d u$ champ électrique sur la cathode ;

- pour un éclairement uniforme de la photocathode, la largeur à mi-hauteur est égale à 575 ps (Fig. 6b), cette valeur est de 450 ps pour un éclairement ponctuel (position 9).

\subsection{FluCtuAtions TEMPORELles LIÉES AU MULTI-} PLICATEUR D'ÉLECTRONS. - De nombreux travaux théoriques $[20,21,22]$ et expérimentaux $[3,23]$ ont été consacrés à l'étude de la résolution en temps des compteurs à scintillations en analysant d'une part les caractéristiques stochastiques du signal anodique de courant, d'autre part les courbes de coïncidences promptes. Les propriétés statistiques de l'amplitude de cette impulsion peuvent être décrites en considérant la fonction d'illumination équivalente $j(t)$ et la réponse percussionnelle $\bar{h}(t)$ du multiplicateur d'électrons (single electron response SER). La fonction de réponse $\bar{h}(t)$ représente la courbe de variation moyenne du courant anodique lorsque un seul photoélectron atteint la première dynode ; $j(t)$ est la densité de probabilité de l'intervalle de temps séparant la date de création de la scintillation et l'instant d'arrivée d'un photoélectron sur la première dynode et on a :

$$
j(t)=i(t) * k(t) .
$$

Sous réserve de validité des hypothèses introduites par Gatti et coll. [24], la valeur moyenne et la variance du signal anodique $S(t)$ lorsque la charge collectée à l'anode est égale à $Q$, sont données par les relations suivantes [22] :

$$
\begin{aligned}
& S(t / Q)=\frac{Q}{G} j(t) * \bar{h}(t) \\
& \sigma_{S}^{2}(t / Q)=\frac{Q}{G}\left(1+\varepsilon_{G}^{2}\right)\left\{j(t) * \bar{h}^{2}(t)-[j(t) * \bar{h}(t)]^{2}\right\}
\end{aligned}
$$

$G$ et $\varepsilon_{G}^{2}$ désignant respectivement le gain du multiplicateur d'électrons et sa variance relative.

En tenant compte de la répartition des amplitudes des impulsions de charge, de valeur moyenne $\bar{Q}$ et d'écart-type $\sigma_{Q}$, les expressions précédentes se mettent sous la forme [22] :

$$
\begin{aligned}
& S(t)= \frac{\bar{Q}}{G} j(t) * \bar{h}(t) \\
& \sigma_{S}^{2}(t)=\frac{\bar{Q}}{G}\left(1+\varepsilon_{G}^{2}\right)\left\{j(t) * \bar{h}^{2}(t)-[j(t) * \bar{h}(t)]^{2}\right\}+ \\
&+\frac{\sigma_{Q}^{2}}{G^{2}}[j(t) * \bar{h}(t)]^{2}
\end{aligned}
$$

Sur la figure 8 sont représentées schématiquement l'impulsion de courant $S(t)$ et les courbes correspondant à une amplitude $S(t) \pm \sigma_{S}(t)$.

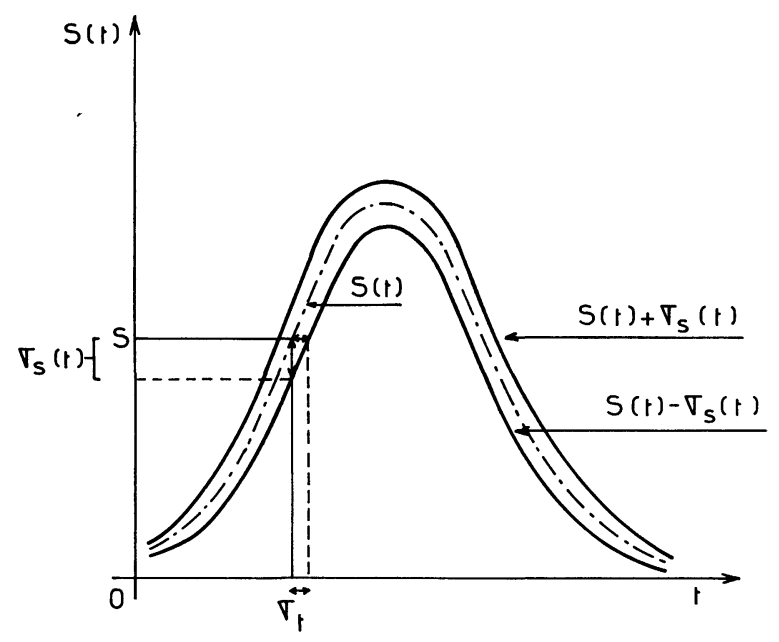

FIG. 8. - Influence des fluctuations d'amplitude du signal anodique sur l'instant de franchissement du seuil $s$.

En général, l'instant d'arrivée de l'impulsion anodique est donné par la date $t$ à laquelle le signal atteint le seuil de déclenchement $s$ d'un discriminateur; un raisonnement géométrique (Fig. 8) indique qu'en première approximation, l'écart-type de $t$ est égal au rapport de celui des hauteurs d'impulsions à la pente du signal, on a :

$$
\begin{aligned}
\sigma_{t}^{2} & =\frac{\sigma_{S}^{2}(t)}{\left(\frac{\mathrm{d} S(t)}{\mathrm{d} t}\right)^{2}} \\
& =\frac{\sigma_{S}^{2}(t / Q)}{\left[\frac{\mathrm{d} S(t)}{\mathrm{d} t}\right]^{2}}+\frac{\sigma_{Q}^{2}}{\bar{Q}^{2}} \frac{S^{2}(t)}{\left[\frac{\mathrm{d} S(t)}{\mathrm{d} t}\right]^{2}} .
\end{aligned}
$$

La variance de l'instant de franchissement du seuil d'un discriminateur est une fonction de la pente du front de montée de l'impulsion anodique ; elle est la somme de deux termes :

- l'un est lié aux fluctuations du signal anodique et résulte de la dispersion des temps de transit des photoélectrons dans l'optique d'entrée et de celle des électrons entre deux dynodes ; 
- l'autre fait intervenir la résolution en amplitude du multiplicateur ; en considérant seulement les variations de gain, on peut établir la relation précédente par un calcul de probabilité (appendice).

La validité de cette expression a été discutée par Donati et coll. [25] à partir d'une étude théorique de la résolution en temps d'un compteur à scintillations comprenant un scintillateur NATON 136 et un photomultiplicateur XP 1020 ; tenant compte d'une solution rigoureuse établie à l'aide d'un ordinateur, ils justifient l'approximation de l'expression précédente dans le cas où le nombre de photoélectrons créés n'est pas trop faible. Sur la figure 9 sont reportées les courbes de

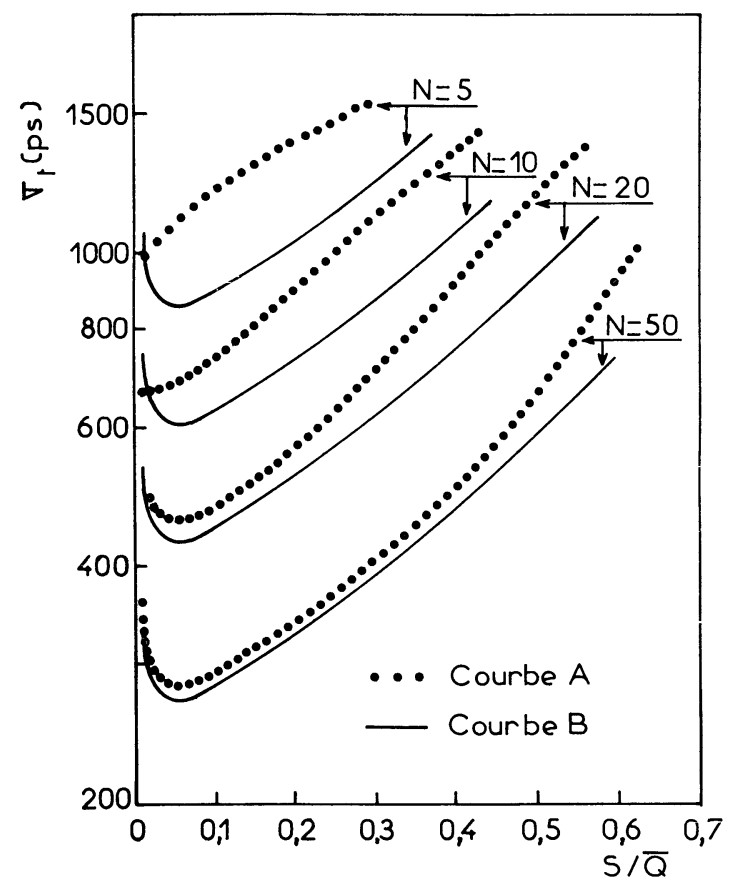

Fig. 9. - Courbes de variation de l'écart-type $\sigma_{t}$ en fonction du seuil $s$ et pour différentes valeurs du nombre de photoélectrons incidents $N$.

variation, en fonction du rapport $S / \bar{Q}$, de l'écart-type calculé par Donati et coll. [25], pour différentes valeurs de la charge collectée à l'anode. Les courbes A correspondent aux résultats déterminés par des méthodes numériques et les courbes $B$ à ceux obtenus à partir de la relation (13) [25]. Il est à noter que des différences significatives entre ces résultats apparaissent lorsque le nombre moyen $N$ de photoélectrons est inférieur à 10.

Les mesures de la résolution en temps des compteurs à scintillations $[3,23]$ sont en bon accord avec les estimations théoriques. Par contre, l'effet de la dispersion temporelle introduite par le multiplicateur dans les expériences de reconstitution d'une impulsion lumineuse n'a pas été jusqu'à présent examiné.

Nous avons repris l'analyse des distributions $r_{1 \exp }(t)$ en fonction du seuil de déclenchement du discriminateur dans le but de préciser l'influence des variations de forme du signal anodique et celle résultant des fluctuations de gain du multiplicateur. La variance de l'instant d'apparition $t$ du signal anodique peut se mettre sous la forme suivante :

$$
\sigma_{t}^{2} \approx \sigma_{t}^{2}(\bar{Q})+\frac{\sigma_{Q}^{2}}{\bar{Q}^{2}}\left[\frac{\bar{h}(t)}{\frac{\mathrm{d} \bar{h}(t)}{\mathrm{d} t}}\right]^{2}
$$

La quantité $\sigma_{t}(\bar{Q})$ est l'écart-type de l'instant $t$ lorsque la charge totale collectée, correspondant à l'émission d'un seul photoélectron, est égale à $\bar{Q}$; en pratique, cette condition peut être obtenue en choisissant à l'aide d'un sélecteur monocanal les amplitudes des signaux anodiques proportionnelles à $\bar{Q}$. Le dernier terme de l'équation précédente représente la dispersion temporelle due aux variations des hauteurs d'impulsions de charge. En supposant que les diverses fluctuations temporelles (optique d'entrée, multiplicateur d'électrons) sont indépendantes, la variance $\sigma_{t}^{2}(\bar{Q})$ est donnée par :

$$
\sigma_{t}^{2}(\bar{Q})=\sigma_{i}^{2}+\sigma_{k}^{2}+\sigma_{m}^{2}(t)
$$

$\sigma_{i}^{2}$ et $\sigma_{k}^{2}$ désignant la variance de l'impulsion lumineuse et celle du temps de transit des photoélectrons; $\sigma_{m}^{2}(t)$ caractérise les fluctuations du multiplicateur, en particulier les variations de forme du signal anodique.

Les histogrammes $r_{1 \exp }(t)$ ont été relevés avec un photomultiplicateur RCA 8850 éclairé par des impulsions brèves (Fig. 2) et dans les conditions suivantes :

- seuils de déclenchement du discriminateur compris entre $20 \mathrm{mV}$ et $200 \mathrm{mV}$;

- amplitude moyenne du signal anodique voisine de $600 \mathrm{mV}$;

- largeur de la fenêtre du sélecteur égale à $5 \%$ de la hauteur moyenne des impulsions de charge ;

— éclairement ponctuel de la photocathode.

Les variations avec le seuil $s$, de la largeur à mihauteur $l_{1 / 2}$ et de l'écart-type $\sigma$ de ces distributions, sont représentées sur la figure $10 a$. On constate que les quantités $l_{1 / 2}$ et $\sigma$ sont des fonctions croissantes du seuil $s$ : ceci vérifie le fait que la variance $\sigma_{m}^{2}(t)$ dépend des fluctuations de forme de l'impulsion anodique de courant. On note en vertu de la relation (16), pour les valeurs extrêmes de $s$, un accroissement $\Delta \sigma_{m}$ de l'écarttype égal à 245 ps.

Sur la même figure sont également reportées, en fonction de $s$, les données expérimentales $l_{1 / 2}^{\prime}$ et $\sigma^{\prime}$ trouvées lorsque la photocathode est uniformément éclairée. On remarque que l'écart-type est pratiquement constant ; ce dernier résultat est compatible avec la valeur $\Delta \sigma_{m}$ calculée précédemment, dans le cas d'un éclairement ponctuel; en effet, si on admet que la variance $\sigma_{m}^{2}(t)$ dépend faiblement de la fonction d'illumination équivalente, on $a$, en tenant compte de l'expression (16) :

$$
\sigma^{\prime}(200 \mathrm{mV})=\sqrt{\sigma^{\prime 2}(20 \mathrm{mV})+\Delta \sigma_{m}^{2}} .
$$




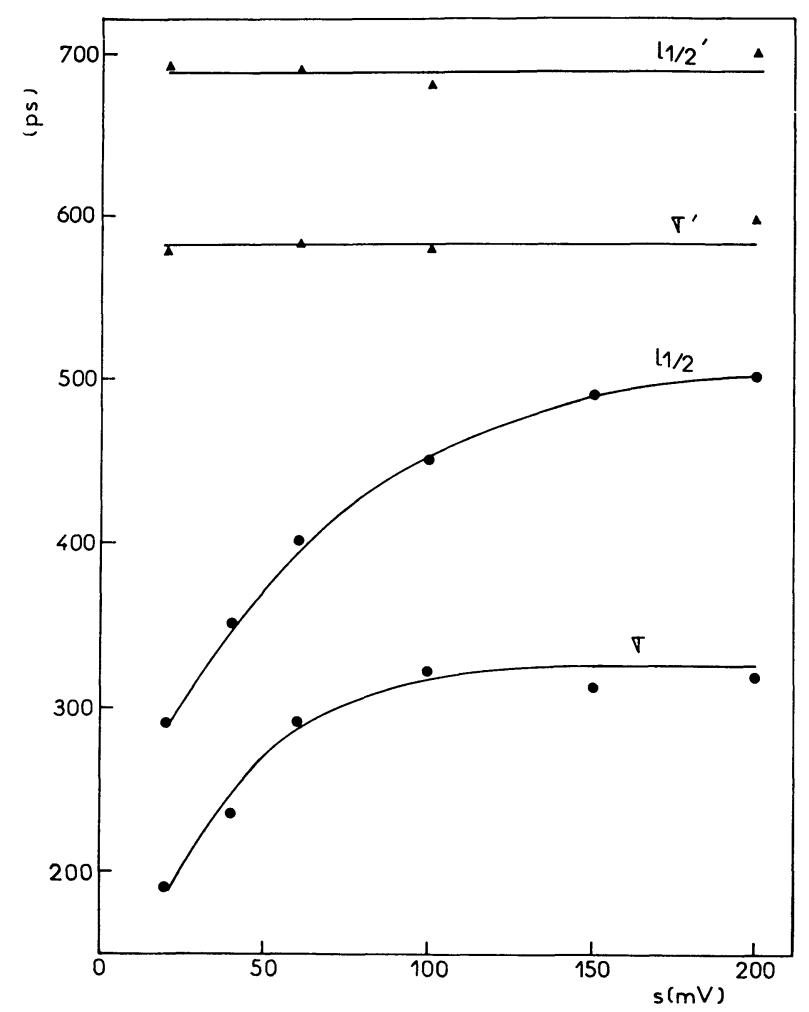

FIG. 10a. - Courbes de variations des largeurs à mi-hauteur $l_{1 / 2}$ et des écart-types $\sigma$ en fonction du seuil $s$ (éclairements ponctuel et uniforme de la photocathode).

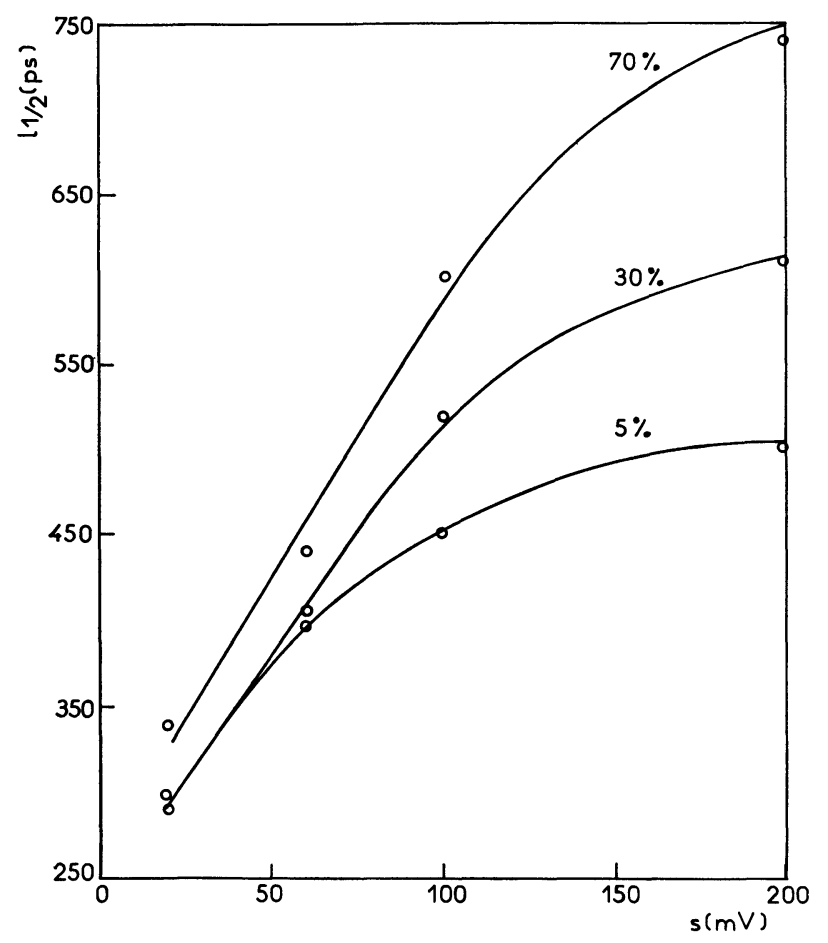

Fig. 10b. - Influence de la dispersion en amplitude du signal anodique sur la largeu $r$ à mi-hauteur des histogrammes $r_{1 \exp }(t)$.

Avec $\sigma^{\prime}(20 \mathrm{mV})=580 \mathrm{ps}$, on en déduit que la valeur correspondant à un seuil de $200 \mathrm{mV}$ est égale à $630 \mathrm{ps}$, résultat en accord avec la donnée expérimentale. On peut conclure que, du fait de l'élargissement de la fonction d'illumination équivalente $i(t) * k(t)$, la contri- bution des fluctuations en temps du multiplicateur est négligeable.

La dispersion temporelle due aux fluctuations d'amplitude des signaux anodiques a été mise en évidence en mesurant les largeurs à mi-hauteur des répartitions $r_{1 \text { exp }}(t)$ pour des largeurs de fenêtre du sélecteur respectivement égales à $5 \%, 30 \%$ et $70 \%$ de la charge moyenne collectée (Fig. 10b) ; remarquons que le spectre des hauteurs d'impulsions en photoélectron unique (Fig. 1) est voisin d'une distribution normale et la résolution en amplitude égale à $30 \%$. De l'examen des données expérimentales, il ressort que, en raison de la faible valeur de la variance relative du gain du multiplicateur $\sigma_{Q}^{2} / Q^{2}=0,014$, l'effet des variations en amplitude (15) est fortement diminué pour des seuils faibles.

4. Conclusion. - L'étude des fluctuations temporelles d'un photomultiplicateur à grand gain de première dynode a été abordée dans le but de déterminer la résolution en temps du compteur utilisé dans les conditions de photoélectrons uniques et en vue de reconstituer des impulsions lumineuses très brèves. Nous avons mis en évidence expérimentalement la contribution respective des dispersions en temps dues à l'optique d'entrée et au multiplicateur d'électrons. Il ressort de ce travail que pour des impulsions lumineuses, de durée inférieure à $250 \mathrm{ps}$, la largeur à mi-hauteur de la distribution $r_{1 \exp }(t)$, augmente lorsque le seuil du discriminateur qui définit l'instant d'apparition du signal anodique croît. Cet effet est attribué, en accord avec les résultats de l'étude des propriétés statistiques des compteurs à scintillations entreprise par Gatti, à l'influence des fluctuations temporelles intervenant dans le multiplicateur et résultant à la fois du processus d'émission secondaire et des variations du temps de transit des électrons entre deux dynodes. Pour des éclairs lumineux, de largeur supérieure à $500 \mathrm{ps,} \mathrm{la}$ contribution précédente à l'élargissement de la courbe de réponse temporelle du détecteur est négligeable. Il résulte également de cette étude que, en raison de l'excellente résolution en amplitude du photomultiplicateur, l'effet des fluctuations des hauteurs des signaux anodiques sur la résolution en temps est sensiblement diminué.

Appendice. - Soit $H(t)$ une impulsion anodique de courant

$$
H(t)=Q h(t)
$$

$Q$ désignant la charge collectée à l'anode et $h(t)$ la réponse percussionnelle du multiplicateur d'électrons.

Si on désigne par $s$ la valeur du seuil de déclenchement du discriminateur, l'instant d'apparition $t$ du signal anodique (Fig. 8) vérifie les équations suivantes :

$$
\begin{aligned}
& s=Q h(t) \\
& t=h^{-1}(s / Q) .
\end{aligned}
$$

La distribution des hauteurs d'impulsions $Q$ peut être caractérisée par la valeur moyenne $Q$ et l'écarttype $\sigma_{Q} ;$ dans ces conditions, on peut montrer que la 
variance de l'instant de franchissement $t$ du seuil $s$ est On en déduit :

donnée par [26]:

$$
\sigma_{t}^{2} \approx \sigma_{Q}^{2}\left[\frac{\mathrm{d} t}{\mathrm{~d} Q}\right]_{Q=\bar{Q}}^{2}
$$

$$
\frac{\mathrm{d} t}{\mathrm{~d} Q}=-\frac{1}{Q^{2}} \frac{s}{\frac{\mathrm{d} h}{\mathrm{~d} t}}=-\frac{1}{Q} \frac{h(t)}{h^{\prime}(t)}
$$

on a :

et

$$
\frac{\mathrm{d}(\mathrm{s} / Q)}{\mathrm{d} Q}=\frac{\mathrm{d} h(t)}{\mathrm{d} t} \cdot \frac{\mathrm{d} t}{\mathrm{~d} Q}
$$

$$
\sigma_{t}^{2} \approx \frac{\sigma_{Q}^{2}}{\bar{Q}^{2}}\left[\frac{h(t)}{h^{\prime}(t)}\right]^{2}
$$

\section{Bibliographie}

[1] Corney, A., Advan. Electron. Electron Physics (Academic Press) Vol. 29 (1970) p. 115.

[2] SCHUYler, R. et Isenberg, I., Rev. Sci. Instrum. 42 (1971) 813.

[3] SipP, B. et Miehe, J. A., Nucl. Instrum. Meth. 144 (1973) 255.

[4] De la Barre, F., Nucl. Instrum. Meth. 102 (1972) 77.

[5] LeSKovar, B. and Lo, C. C., IEEE Trans. Nucl. Sci. NS-19 (1972) 3, 50.

[6] SCARL, D. B., IEEE Trans. Nucl. Sci. NS-19 (1972) 7.

[7] BROLL, N., à paraître.

[8] Poultney, S. K., Advan. Electron. Electron Physics (Academic Press) Vol. 31 (1972) p. 39.

[9] Cova, S., Bertolaccini, M. and Bussolati, C., Phys. Stat. Sol. (a) 18 (1973) 4.

[10] Miehe, J. A., Ambard, G., Zampach, J. et Coche, A., IEEE Trans. Nucl. Sci. NS-17 (1970) 115.

[11] Lehmberg, R. H., Phys. Rev. (1968) 1152.

[12] Rousseau, M., J. Physique 30 (1969) 675.
[13] Bollinger, L. M. et Thomas, G. E., Rev. Sci. Instrum. 32 (1961) 1044.

[14] Koechlin, Y., thèse, Paris (1961)

[15] Pfeffer, thèse, Strasbourg (1965).

[16] PIETRI, G., Acta Electron. 5 (1961) 7.

[17] BARBIER, M., Acta Electron. 5 (1961) 31.

[18] Miehe, J. A. et KNISPel, G., C. R. Hebd. Séan. Acad. Sci. 273B (1971) 285.

[19] SIPP, B. et MIEHE, J. A., Nucl. Instrum. Meth 144 (1973) 249.

[20] Gatti, E. et Svelto, V., Nucl. Instrum. Meth. 30 (1964) 231.

[21] Euling, R., J. Appl. Phys. 35 (1964) 1391.

[22] Bertolaccini, M., Bussolati, C., Cova, S., Donati, S. et Svelto, V., Nucl. Instrum. Meth. 51 (1967) 325.

[23] Bengtson, B. et Moszynski, M., Nucl. Instrum. Meth. 81 (1970) 109.

[24] Gatti, E. et Svelto, V., Nucl. Instrum. Meth. 43 (1966) 248

[25] Donati, S., Gatti, E. and Svelto, V., Nucl. Instrum. Meth. 77 (1970) 179.

[26] Papoulis, A., Random Var. and Stoch. Processes (Mc GrawHill) (1965). 